\title{
CrystEngComm
}

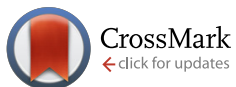

Cite this: CrystEngComm, 2015, 17, 463

Received 21st July 2014,

Accepted 7th October 2014

DOI: $10.1039 / c 4 c e 01512 f$

www.rsc.org/crystengcomm

\section{Surfactant-directed syntheses of mesostructured zinc imidazolates: formation mechanism and structural insights $\uparrow$}

\author{
E. A. Flügel, ${ }^{a b}$ M. T. Aronson, ${ }^{d}$ S. C. Junggeburth, ${ }^{a b c}$ B. F. Chmelka ${ }^{d}$ \\ and B. V. Lotsch*abc
}

\begin{abstract}
Supramolecular templating techniques have been widely used to direct the formation of porous materials with the goal of introducing permanent mesoporosity. While surfactant-directed self-assembly has been exploited for inorganic materials such as titania, silica, organosilica, and zeolites, it has rarely been applied to metal-organic frameworks (MOFs) and coordination polymers. Here we introduce a new family of gemini surfactant-directed zinc imidazolates, referred to as mesostructured imidazolate frameworks (MIFs), and present a detailed study on the influence of different gemini-type surfactants on the formation mechanism and structures of the resulting zinc imidazolates. The proposed formation mechanism for MIF-type materials involves co-assembly and crystallization processes that yield lamellar mesostructured imidazolate frameworks. Understanding and controlling such processes also has implications for the syntheses of microporous zinc imidazolate framework (ZIF) materials, whose formation can be suppressed in surfactant-rich solutions, whereas formation of MIF materials is favored in the presence of surfactants and triggered by the addition of halogenides. Solid-state $2 \mathrm{D}{ }^{13} \mathrm{C}\left\{{ }^{1} \mathrm{H}\right\} \mathrm{HETCOR}$ NMR measurements on prototypic CTAB-directed MIF-1 establish that the head group moieties of the surfactant molecules interact strongly with the zinc-imidazolate-bromide sheets. Additionally, the NMR analyses suggest that MIF-1 has a significant fraction of surfactant molecules that are interdigitated between the zinc-imidazolate-bromide sheets with an antiparallel stacking arrangement, consistent with the high thermal and chemical stability of the MIF hybrid materials.
\end{abstract}

\section{Introduction}

In recent years, metal-organic frameworks (MOFs) have received considerable attention due to their modularity and versatility, such as tunable pore sizes, ${ }^{1,2}$ high specific surface areas ${ }^{3-5}$ and the possibility to alter their properties via post-synthetic modification of the organic linkers. ${ }^{6,7}$ These desirable properties render MOFs multi-purpose scaffolds in a number of applications such as catalysis, ${ }^{8,9}$ sensing, ${ }^{10,11}$ gas storage ${ }^{12,13}$ and drug delivery. ${ }^{14,15}$ Limitations in the use of MOFs in catalysis mainly arise from a lack of chemical stability as well as small pore sizes in the micropore regime, as only a limited number of MOFs with pore sizes larger than $2 \mathrm{~nm}$ have been

\footnotetext{
${ }^{a}$ Max Planck Institute for Solid State Research, Stuttgart, Germany.

E-mail: b.lotsch@fkf.mpg.de

${ }^{b}$ Ludwig Maximilian University (LMU), Department of Chemistry, Munich, Germany ${ }^{c}$ Nanosystems Initiative Munich (NIM) \& Center for Nanoscience (CeNS), Munich, Germany

${ }^{d}$ Department of Chemical Engineering, University of California, Santa Barbara, California, USA

$\dagger$ Electronic supplementary information (ESI) available: DTA/TG, IR, PXRD measurements, formation mechanism and structural graphics. See DOI: 10.1039/c4ce01512f
}

reported to date. ${ }^{16-18}$ With interpenetration effects limiting the possibility of using larger linkers to increase the pore size, other routes such as the use of more complex connection motifs ${ }^{17}$ or templating techniques have been utilized. ${ }^{19}$ The latter provide additional benefits by creating a hierarchical structure with bi- or multimodal pore-size distributions, including both micro- and mesopores, which facilitate the diffusion of bulky molecules and widen the potential applications in catalysis and drug delivery.

While exploring synthetic approaches to hierarchical ZIFs by soft templating, we have recently discovered the class of mesostructured imidazolate frameworks (MIFs), which combine structural elements of both coordination polymers and liquid crystalline mesophases to form hierarchical structures ordered on both the atomic- and nanoscale. ${ }^{20}$ MIFs are composed of zinc imidazolate chains or layers sandwiched between surfactant slabs, forming a mesoscopic layered hybrid structure similar to MCM-type lamellar silica or metal oxide mesophases. $^{20,21}$ Therefore, the well-known structural analogies between zeolitic imidazolate frameworks - a subclass of metalorganic frameworks - and zeolites can also be extended mesophase materials, where surfactant-directed lamellar zinc imidazolate 
mesophases find their analogue in MCM-50-type silica mesostructures (Fig. 1a). Lamellar MIFs have previously been obtained under reverse microemulsion conditions in the presence of the surfactant cetyltrimethylammonium bromide (CTAB). Given the structural similarities between MIFs and MCM-50 - both on the molecular level ( $\mathrm{Zn}-\mathrm{Im}-\mathrm{Zn}$ vs. Si-O-Si bonding angles) and on the mesoscale (mesoscopic phase segregation into organic and inorganic lamellar domains) - design principles associated with mesostructured silica materials may ultimately enable the syntheses of MIFs with non-lamellar 3D hexagonal or cubic mesostructures.

Previous work on mesostructured silica materials has revealed the dominant role of the surfactant and its amphiphilic architecture and colloidal properties on the resulting mesostructures. Ryoo et al. reported the synthesis of a hierarchically nanoand mesoporous MFI zeolite, where gemini-type surfactants directed the formation of MFI nanosheets with a multilamellar structure. $^{22-24}$ Gemini surfactants can be described by the general structure $n-m-n$, with $n$ being a terminal aliphatic carbon chain and $m$ being a linker between two quaternary nitrogen ions (Fig. 1b). Importantly, surfactant properties such as packing parameter and critical micelle concentration, which ultimately determine the micellar curvature, are a function of the size, geometry and charge density of the gemini surfactant molecules. $^{25-28}$ Earlier work on the influence of these surfactants on the formation of periodic mesoporous organosilica (PMO) has shown that $n-6-n$ gemini surfactants ( $n=12-22$ ) can form multiple mesostructures, depending on the length of the terminal carbon chains. For example, shorter chains $(n=12-14)$ result in cubic phases, while longer chains often lead to $3 \mathrm{D}$ and $2 \mathrm{D}$ hexagonal $(n=16-20)$ or lamellar $(n=22)$ structures. $^{29}$ In this work, we use gemini surfactants with different lengths of their alkyl chains (from 14 to 18 carbon atoms) and spacer lengths (4 to 10 carbon atoms) to synthesize a homologous series of mesostructured imidazolate frameworks. To develop a better understanding of the mechanisms underlying MIF formation, we rely on a combination of spectroscopic analyses that provide complementary information across multiple length scales. In particular, two-dimensional (2D) solid-state nuclear magnetic resonance (NMR) spectra establish specific surfactant-framework interactions in zinc methylimidazolate CTAB hybrid materials. The detailed insights provided by this work are expected to aid in the design of MIFs with a broader

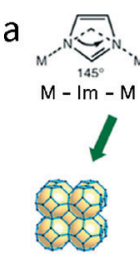

Zeolite A / ZIF-8

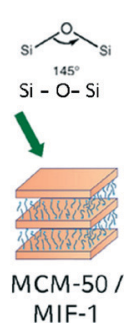

b

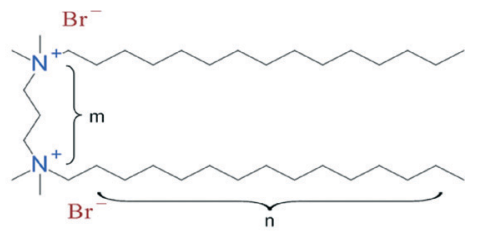

Fig. 1 (a) Schematic diagram showing the similarities between ZIFs and zeolites on the one hand, and MIFs and MCM-type materials on the other hand. (b) Molecular structure of a representative gemini surfactant (here $n=15, m=3$ ). The spacer length $m$ and the carbon tail length $n$ can be altered to tune the properties of the surfactant. range of mesostructured compositions, topologies, and hierarchical structural ordering.

\section{Experimental section}

\subsection{Reagents \& instruments}

All reagents were commercially available and used as purchased without further purification.

Powder X-ray diffraction (PXRD) measurements were performed using a STOE Stadi $P$ powder X-ray diffraction instrument, operated in Guinier geometry using a Mythen $1 \mathrm{~K}$ or an imaging plate detector. Infrared measurements were performed on a Perkin Elmer Spektrum BX II with an attenuated total reflectance unit. Elemental analysis was done in a thermal conductivity measurement cell of an Elementar vario EL. Analysis of halogenide content was done by titration with $\mathrm{Ag}\left(\mathrm{NO}_{3}\right)$ on a Titroprocessor 672 by Metrohm. To determine the amount of zinc and other metal ions, the samples were analyzed based on optical emission of the elements in an Ar-plasma (ICP). Measurements were done on a VARIAN VISTA simultaneous spectrometer with autosampler and recorded with a CCD-detector. TEM measurements were performed on a Philips CM 30 ST microscope $\left(\mathrm{LaB}_{6}\right.$ cathode, $\left.300 \mathrm{kV}, C_{\mathrm{S}}=1.15 \mathrm{~mm}\right)$. Sample preparation was done by drop casting highly diluted samples $\left(\sim 50 \mu \mathrm{g} \mathrm{mL}^{-1}\right)$ on a carbon coated copper grid, followed by evaporation of the solvent. Solid-state NMR experiments were conducted at 11.7 T using Bruker AVANCE II and DSX-500 NMR spectrometers, both operating at frequencies of $500.24 \mathrm{MHz}$ for ${ }^{1} \mathrm{H}$, 125.78 MHz for ${ }^{13} \mathrm{C}$, and $50.70 \mathrm{MHz}$ for ${ }^{15} \mathrm{~N}$. The experiments were performed at room temperature under magic-angle-spinning (MAS) conditions using a $4 \mathrm{~mm}$ Bruker $\mathrm{H}$-X double-resonance probehead and zirconia rotors. For the solid-state $2 \mathrm{D}{ }^{13} \mathrm{C}\left\{{ }^{1} \mathrm{H}\right\}$ HETeronuclear CORrelation (HETCOR) NMR experiments, high-power homonuclear ${ }^{1} \mathrm{H}-{ }^{1} \mathrm{H}$ decoupling was applied during the ${ }^{1} \mathrm{H}$ evolution period to enhance the resolution in the ${ }^{1} \mathrm{H}$ dimension, using the eDUMBO- 1 pulse sequence ${ }^{30}$ with a phase-modulated radio frequency pulse of constant amplitude (100 kHz). The 2D ${ }^{13} \mathrm{C}\left\{{ }^{1} \mathrm{H}\right\}$ HETCOR spectra were acquired with cross-polarization (CP) contact times of $1 \mathrm{~ms}$ or $5 \mathrm{~ms}$, a recycle delay of $1 \mathrm{~s}$, and SPINAL-64 ${ }^{1} \mathrm{H}$ heteronuclear decoupling (80 kHz). 128 transients $\left(t_{2}\right)$ were signal-averaged for each of the $400 t_{1}$ increments, resulting in a total experimental time of approximately $14 \mathrm{~h}$ for each spectrum. Thermogravimetric and differential thermal analysis measurements were performed on a combined DTA-TG-thermobalance (type 92-2400, Setaram). The samples were transferred into an aluminum oxide crucible and heated from room temperature to $700{ }^{\circ} \mathrm{C}$ at a rate of $5^{\circ} \mathrm{C} \min ^{-1}$.

\subsection{Synthesis of gemini surfactants}

The gemini surfactants were synthesized by dissolving $3 \mathrm{mmol}$ of the respective $N, N$-dimethylamine in $75 \mathrm{~mL}$ of acetone and adding $1.5 \mathrm{mmol}$ of the respective $\alpha, \omega$-dibromoalkane to the solution (for details on all chemicals used see Table S1 $\dagger$ ). The solution was refluxed for 4 days, leading to the formation of a white precipitate that was obtained by centrifugation at 
$15000 \mathrm{rpm}$ for 10 minutes. The product was dried at room temperature overnight and yielded a white, waxy substance. Purity of the obtained surfactants was confirmed by elemental analysis, IR spectroscopy and solution-state NMR.

\subsection{Synthesis of mesostructured imidazolate frameworks}

In a typical synthesis, $1 \mathrm{mmol}$ of surfactant was dissolved in $25 \mathrm{~mL}$ of solvent (water, methanol, ethanol, 2-propanol, THF, aceton, ethylacetate, diethylether, dioxan or $n$-heptane/1-hexanol (10:1)). $0.603 \mathrm{mmol}\left(603 \mu \mathrm{L}, 1 \mathrm{~mol} \mathrm{~L}^{-1}\right.$ in water) zinc acetate and $0.603 \mathrm{mmol}\left(335 \mu \mathrm{L}, 1.8 \mathrm{~mol} \mathrm{~L}^{-1}\right.$ in water) of (methyl) imidazole were added to the solution and the resulting mixture was stirred overnight at room temperature. The formed precipitate was collected by centrifugation (20.000 rpm, $5 \mathrm{~min}$ ), washed with 2-propanol and dried at room temperature.

\section{Results and discussion}

MIFs were obtained in the presence of $n-m-n$ gemini surfactants ( $n=14-18, m=4-10$, see Fig. $1 b)$ as structure directing agents in a variety of solvents ranging from methanol to inverse microemulsion systems such as $n$-heptane/1-hexanol/ water mixtures and THF. The materials could be obtained with imidazole (IM) and 2-methylimidazole (MeIM) as linkers, at room temperature as well as under reflux conditions. In order to obtain differently mesostructured materials, the ratio of IM to $\mathrm{Zn}\left(\mathrm{NO}_{3}\right)_{2}$ was altered between $8: 1$ and $1: 4$ and the surfactant concentration was varied between $0.01 \mathrm{M}$ and $0.5 \mathrm{M}$. Although $n-m-n$ gemini surfactants were reported to direct the formation of 3D cubic ( $n=16-18, m=10-12)$ and hexagonal ( $n=16-18, m=6-10$ ) mesostructures in the MCM-type system, ${ }^{31}$ we found the signature of lamellar MIF mesophases in all cases, irrespective of the type of gemini surfactant/solvent combination used, over the whole range of surfactant concentrations tested. The stacking parameters varied slightly depending on the solvent used (Table S2, Fig. S1 $\dagger$ ), yet could not be correlated to specific solvent properties such as boiling point or dielectric constant.

The obtained MIF phases exhibited varying degrees of crystallinity, and only a small fraction of the materials yielded powder patterns of suitable quality for indexing. Pawley refinement of the XRD pattern of 16-8-16 imidazolate MIF yielded metrics consistent with monoclinic symmetry $(C 2, a=11.5829 \AA$, $b=58.253 \AA, c=9.2415 \AA, \beta=88.94^{\circ}, \mathrm{w} R=7.57, \mathrm{GoF}=1.286$, Fig. S2 $\dagger$ ). Similar metrics were determined for gemini-directed MIFs with $m \leq 8$. Although all samples are highly sensitive to the electron beam, transmission electron microscopy selected area diffraction (TEM SAED) data obtained for the 16-10-16 sample revealed a rectangular pattern with $a=11.5 \AA$ and $c=9.2 \AA$ and a lamellar stacking of $29 \AA$ along [0k0] (Fig. 2), which is in principle agreement with the powder data. The doubled stacking parameter along $b$ that can be observed in the Pawley fit may be rationalized by a superstructure, which prominently was observed for MIFs based on gemini-surfactants with $m \leq 8$. The absence of higher order stacking reflections at around

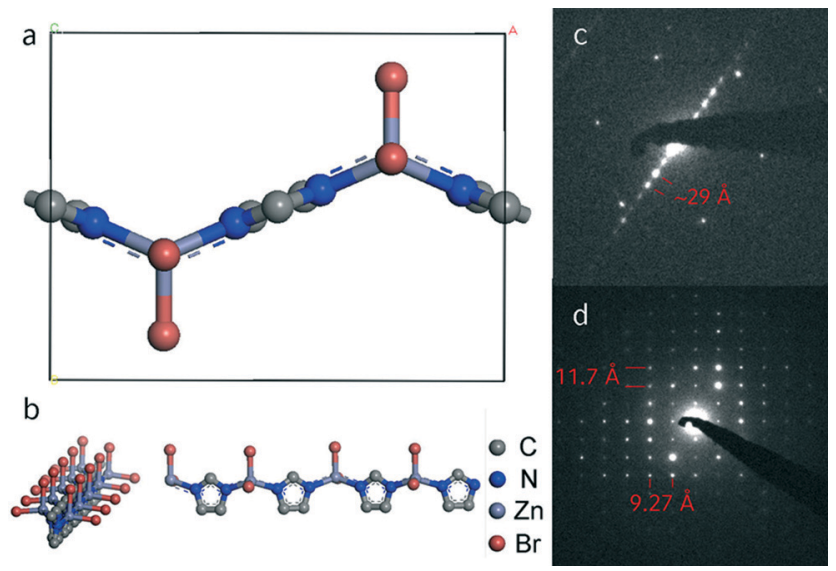

Fig. 2 (a) Structure model of the zinc imidazolate bromide chain viewed along the $b$-axis and (b) along/onto the chains. (c, d) TEM SAED images of 16-6-16 IM MIF (c) perpendicular to the [010] direction and (d) of the (101) plane.

$2 \theta=5^{\circ}$ in hybrid materials with shorter linkers may be due to the discussed superstructure effects or accidental extinction. ${ }^{22,32}$

Elemental analysis revealed a composition of all hybrid materials of zinc: IM/MeIM : bromine : surfactant of $1: 1: 2: 0.5$, corresponding for example to the empirical formula $\mathrm{Zn}_{2} \mathrm{Br}_{4}\left(\mathrm{C}_{3} \mathrm{H}_{3} \mathrm{~N}_{2}\right)_{2}\left(\mathrm{C}_{46} \mathrm{H}_{96} \mathrm{~N}_{2}\right)$ for the 16-10-16 imidazolate MIF (Table $\mathrm{S} 3 \dagger$ ). The synthesized materials show good thermal stability up to $\approx 320^{\circ} \mathrm{C}$ (Fig. S3†), as well as chemical stability, withstanding even surfactant extraction procedures with supercritical $\mathrm{CO}_{2}$, yet showing the typical sensitivity of MOFs towards acids. Upon acid-induced decomposition of the material, the pristine surfactants and imidazolates were recovered, proving that all MIF constituents stay intact during the entire synthesis process.

Our data therefore suggest that all gemini-directed materials are composed of the same principal building units like the prototypic MIF-1, ${ }^{20}$ as sketched in Fig. 2 . The formerly proposed structure model consists of one-dimensional zinc-imidazolate chains with additional coordination of two bromine ions to the metal center. These chains of corner sharing tetrahedra are interleaved with surfactant layers, thus forming quasi twodimensional layered assemblies. This structural motif for the zinc imidazolate subunit has previously been reported by Lin et al. in a coordination polymer without incorporated surfactant molecules. ${ }^{33,34}$ Combination of the developed structure model with the metrics obtained from the Pawley fit shows good accordance between both (Fig. 2).

A solid-state ${ }^{13} \mathrm{C}$ MAS NMR spectrum of the 16-10-16 MeIM MIF material (Fig. 3b) exhibits two distinct high-frequency signals for MeIM ( $\delta=149.4,125.1 \mathrm{ppm}$ for C3-C1), similar to the ${ }^{13} \mathrm{C}$ signals observed for ZIF-8 $(\delta=150.0,125.2 \mathrm{ppm}) \cdot{ }^{20}$ The ${ }^{13} \mathrm{C}$ signals corresponding to the surfactant $(\delta=67.1$, $48.2,43.0,32.0,29.8,26.3,23.0,15.8,14.8 \mathrm{ppm})$, as well as those for MeIM are in good agreement with the ${ }^{13} \mathrm{C}$ signals previously reported for MIF-1 (Fig. 3a). ${ }^{20}$ The solid-state ${ }^{15} \mathrm{~N}$ MAS NMR spectrum of the 16-10-16 MeIM MIF (Fig. 3d) 

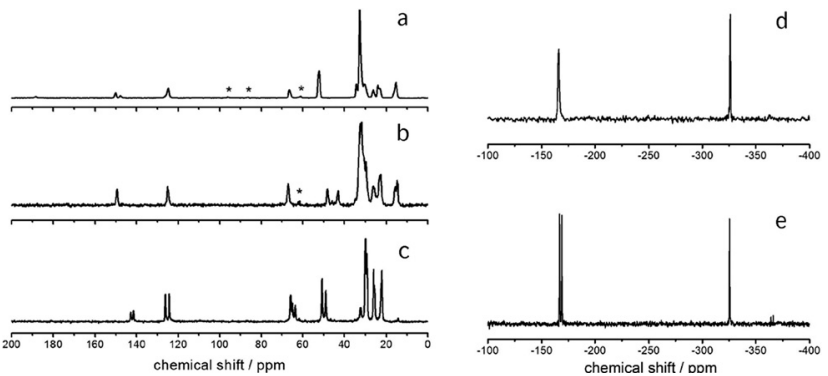

Fig. 3 Solid-state ${ }^{13} \mathrm{C}$ MAS NMR spectra of (a) MIF-1, ${ }^{20}$ (b) $16-10-16$ MeIM MIF and (c) 14-6-14 IM MIF and solid-state ${ }^{15} \mathrm{~N}$ MAS NMR spectra of (d) 16-10-16 MeIM MIF and (e) 14-6-14 IM MIF. Asterisks indicate spinning side bands.

exhibits two distinct ${ }^{15} \mathrm{~N}$ signals associated with the surfactant head groups $(-323.5 \mathrm{ppm})$ and the methylimidazolate ion $(-168.4 \mathrm{ppm})$, respectively, consistent with similar local environments for both types of ammonium groups and both imidazolate nitrogen atoms.

For the 14-6-14 IM MIF material, additional signals are observed in the ${ }^{13} \mathrm{C}$ MAS NMR spectrum, revealing the presence of different ${ }^{13} \mathrm{C}$ local environments. As shown in Fig. 3c, two different pairs of well-resolved ${ }^{13} \mathrm{C}$ signals are observed for the imidazolate carbon atoms (143.1 and $141.6 \mathrm{ppm}$, 126.1 and $124.4 \mathrm{ppm})$. These distinct pairs of ${ }^{13} \mathrm{C}$ signal intensities are consistent with two inequivalent ${ }^{13} \mathrm{C}$ local environments for the imidazolate species, likely due to superstructure effects, such as ABA-type stacking of the surfactantinorganic slabs. Such a stacking arrangement would also give rise to a doubled $b$-axis as found for other hybrid materials ${ }^{35}$ and for pure CTAB. ${ }^{36}$ Interestingly, similar experiments on CTAB-directed MIF-1 (Fig. 3a) also exhibit two distinct ${ }^{13} \mathrm{C}$ signals (149.0 and $151.0 \mathrm{ppm}$ ) for the $\mathrm{sp}^{2}$ carbon atom bonded to the nitrogen atoms of the methylimidazole moieties. These results, along with a detailed 2D NMR analysis of MIF-1, are discussed below.

Subtle but distinct differences are also observed in the ${ }^{15} \mathrm{~N}$ local environments of the 14-6-14 IM MIF, as evidenced by ${ }^{15}$ N MAS NMR (Fig. 3e) and consistent with the ${ }^{13} \mathrm{C}$ NMR analyses above. Two well-resolved and equally intense ${ }^{15} \mathrm{~N}$ signals associated with the imidazolate nitrogen atoms are observed at -166.4 and $-168.9 \mathrm{ppm}$ for the 14-6-14 IM MIF, whereas only one corresponding ${ }^{15} \mathrm{~N}$ signal $(-166.2 \mathrm{ppm})$ is observed for the 16-10-16 MeIM MIF (Fig. 3d). These results indicate that the local environments of the imidazolate species are highly sensitive to surfactant architecture, such as spacer length, leading to inequivalent imidazolate and surfactant interactions predominantly for materials synthesized using surfactants with shorter spacers $(m \leq 8)$.

The gemini MIFs obtained by syntheses with $n-m-n$ gemini surfactants $(n=14,16,18 ; m=4-10)$ all exhibit similar layered zinc-imidazolate structures separated by surfactant molecules, while the stacking parameter of the mesostructures differs for the surfactants used. All synthesized materials exhibit a lamellar mesostructure, with layer stacking distances varying between $25.4 \AA$ and $35.6 \AA$ for IM bridged systems and between $25.3 \AA$ and $35.9 \AA$ for MeIM bridged systems, respectively, depending on the used surfactants (Fig. 4). We find that the stacking of the material depends on both the surfactant tail length $(n)$ as well as on the length of the spacer $(m)$, indicating a tilted arrangement of the surfactants with respect to the zinc-imidazolate slabs. Our data show a direct correlation between the $d$-spacing and the tail length, with an increase in $d$-spacing by $1.7 \pm 0.3 \AA$ for an increase of the tail length by two carbon atoms (Fig. 4, S4 and Table S4†). The observable lamellar stacking also increases with longer spacer lengths, but by a variable amount depending on the used spacer. Whilst the stacking distance increases by $4.6 \AA$ 皮 when switching from $m=9$ to $m=10$ in $18-m-18$ IM MIFs, the lamellar stacking decreases by $\approx 0.1 \AA$ when switching from $m=7$ to $m=8$ in the same MIF system.

A closer look at the proposed structure of the MIF materials provides an explanation for this phenomenon. The distance between two $\left[\mathrm{ZnBr}_{2}\right]$-units is roughly $6 \AA$, which is in good agreement with the distance between the two quartenary nitrogen ion headgroups of a $m=5$ gemini surfactant. Up to a linker length of $m=9$, we see that the surfactants appear to gradually tilt further away from the neighbouring $\left[\mathrm{Zn}(\mathrm{Me}) \mathrm{IMBr}_{2}\right]^{-}$-units in order to comply with the charge density of the inorganic slabs (Scheme S5 $\uparrow$ ). The sudden increase in stacking distance at $m=10$ for IM (Fig. 4, left) is likely accompanied by a more abrupt structural rearrangement, such as an increase in the tilt angle $\alpha$, leading to a more perpendicular alignment of the surfactants with respect to the layers and, hence, increased $d$-spacing.

The tilted arrangement of the gemini surfactants seems contradictory to our previous observations, as this asymmetric arrangement of the surfactants is expected to manifest distinct ${ }^{15} \mathrm{~N}$ NMR signals for the two nitrogen atoms of the gemini head groups due to differences in local environments of the two alkylammonium ions. However, the alkyl-groups surrounding
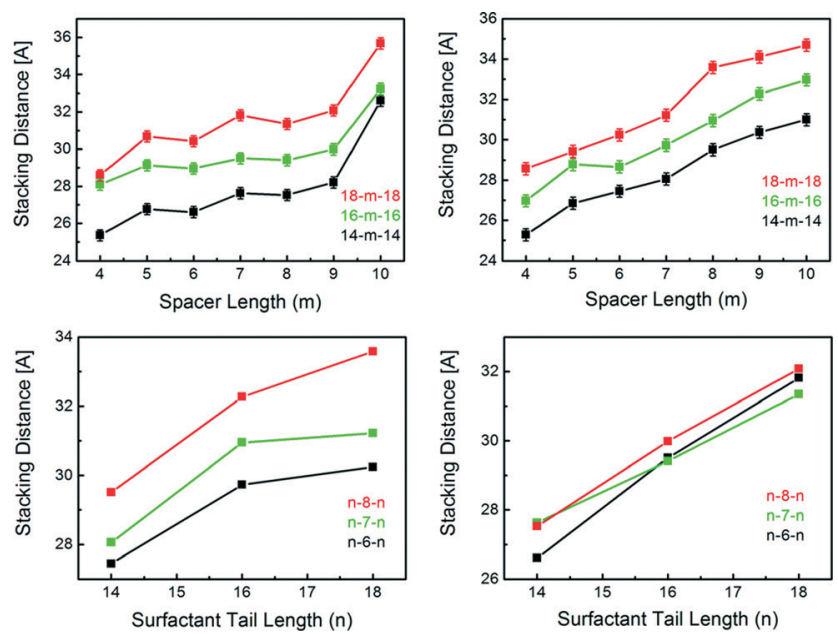

Fig. 4 Graphical presentation of the $d$-values corresponding to the first observable stacking reflection in the PXRD measurements of all synthesized MIF materials as a function of spacer length (top) and tail length (bottom). Left: IM bridged MIFs, right: MelM bridged MIFs. 
the nitrogen atoms may well "screen" them and provide an overall similar local environment, rendering them less susceptible to direct interactions with the coordination polymer chains.

To complete our structural model of gemini-directed MIFs, we assume that the two surfactant carbon tails are oriented in a close-to-parallel conformation, in agreement with previous work on gemini-surfactants with spacer sizes $m>3 .{ }^{37}$ This leads us to propose a model of the surfactant location in the material with both carbon tails parallel to each other with a fixed angle $\alpha$ of about $43^{\circ}$ between the surfactant tail and the zinc imidazolate layers (Fig. S6†).

Previously synthesized prototypic MIFs differ from the materials presented in this work only with respect to the type of surfactant used (alkyl ammonium vs. gemini alkyl ammonium), the resulting $d$-spacings and the apparent structural order, as indicated by the presence of high-angle reflections in the PXRD data of the gemini surfactants as opposed to those of CTAB-directed MIF-1. Despite their similar composition (ratio of inorganic and organic building blocks) and mesostructure, we assume a more rigid anchoring of the doubly cationic ammonium head groups of the gemini surfactants to the inorganic slabs as compared to CTAB-directed MIFs, stabilized by multiple hydrophobic interactions of the antiparallel interdigitated surfactant layers. ${ }^{38}$

To gain additional insights about the locations and interactions of the surfactants within MIF-type materials, solidstate $2 \mathrm{D}{ }^{13} \mathrm{C}\left\{{ }^{1} \mathrm{H}\right\}$ HETeronuclear CORrelation (HETCOR) NMR measurements were performed on a CTAB-directed MIF material (MIF-1). MIF-1 was used instead of the gemini-directed MIFs because the CTAB-based system is less complicated and exhibits similar molecular-level interactions between different organic and inorganic moieties. Interactions among chemically distinct species in heterogeneous zinc imidazolate materials can be established by solid-state 2D NMR techniques that are sensitive to dipole-dipole couplings between molecularly proximate moieties. Here, solid-state $2 \mathrm{D}{ }^{13} \mathrm{C}\left\{{ }^{1} \mathrm{H}\right\}$ HETCOR NMR spectra provide specific local and molecular-level information on $\mathrm{CTA}^{+}$-framework interactions in as-synthesized MIF-1. Furthermore, the intra- and intermolecular interactions are be distinguished by experimentally varying the ${ }^{13} \mathrm{C}-{ }^{1} \mathrm{H}$ cross-polarization contact time from short (e.g., $1 \mathrm{~ms}$ ) to long (e.g., $5 \mathrm{~ms})$ durations, respectively.

The solid-state $2 \mathrm{D}{ }^{13} \mathrm{C}\left\{{ }^{1} \mathrm{H}\right\}$ HETCOR NMR spectra in Fig. 5a of as-synthesized MIF-1 exhibit strong and distinct intensity correlations between ${ }^{13} \mathrm{C}$ and ${ }^{1} \mathrm{H}$ nuclei associated with the $\mathrm{CTA}^{+}$surfactant molecules and the zinc-imidazolate-bromide sheets. A 1D ${ }^{13} \mathrm{C}\left\{{ }^{1} \mathrm{H}\right\}$ CPMAS NMR spectrum is shown above the horizontal axis, along with ${ }^{13} \mathrm{C}$ signal assignments corresponding to the various ${ }^{13} \mathrm{C}$ species of the $\mathrm{CTA}^{+}$surfactant and the methylimidazole moieties. Two 2D NMR spectra conducted with short (1 ms) and long ( $5 \mathrm{~ms}$ ) CP contact times are shown in red and blue, respectively, for comparison. The $2 \mathrm{D}$ signal intensities observed for the short contact time arise from strongly dipolar-coupled nuclei that are associated principally with intramolecular interactions from covalently bonded ${ }^{13} \mathrm{C}$ and ${ }^{1} \mathrm{H}$ nuclei. Strong intensity correlations are observed between the
${ }^{13} \mathrm{C}$ signal of the methylimidazole aromatic carbon atoms $\left(C_{1} / C_{2}\right)$ at $126 \mathrm{ppm}$ and the ${ }^{1} \mathrm{H}$ signals at $6.6 \mathrm{ppm}$ and $7.3 \mathrm{ppm}$ corresponding to their aromatic protons, as expected. Similarly, correlated signal intensity is observed at $16 \mathrm{ppm}\left(C_{17^{\prime}}\right), 17 \mathrm{ppm}$ $\left(C_{4}\right)$, and $53 \mathrm{ppm}\left(C_{1^{\prime}}\right)$ in the ${ }^{13} \mathrm{C}$ dimension associated with distinct methyl groups at $1.3 \mathrm{ppm}, 2.55 \mathrm{ppm}$, and $2.45 \mathrm{ppm}$ in the ${ }^{1} \mathrm{H}$ dimension from their respective covalently bonded hydrogen atoms. Additionally, 2D intensity correlations are observed at $67 \mathrm{ppm}\left(C_{2^{\prime}}\right)$ and $25-35 \mathrm{ppm}\left(C_{3^{\prime}}-C_{16^{\prime}}\right)$ from alkyl chain ${ }^{13} \mathrm{C}$ atoms with the ${ }^{1} \mathrm{H}$ signals at $3.35 \mathrm{ppm}$ and $1.5 \mathrm{ppm}$, associated with the alkyl protons of the $\mathrm{CTA}^{+}$molecules. Together, these intramolecular $2 \mathrm{D}$ intensity correlations allow the numerous partially resolved ${ }^{13} \mathrm{C}$ and ${ }^{1} \mathrm{H}$ signals to be unambiguously assigned to as-synthesized MIF-1.

More interestingly, correlated signal intensity from intermolecular interactions between ${ }^{13} \mathrm{C}$ and ${ }^{1} \mathrm{H}$ nuclei on different molecular species provide complementary information concerning the proximities of the $\mathrm{CTA}^{+}$surfactant and the methylimidazole framework moieties. Correlated signal intensity is observed for a short cross-polarization contact time ( $1 \mathrm{~ms}$, Fig. $5 \mathrm{a}$, red) at $17 \mathrm{ppm}\left(C_{4}\right)$ and $126 \mathrm{ppm}\left(C_{1} / C_{2}\right)$ in the ${ }^{13} \mathrm{C}$ dimension from the anionic zinc methylimidazolate chains and at $2.45 \mathrm{ppm}$ in the ${ }^{1} \mathrm{H}$ dimension associated with the cationic $\mathrm{CTA}^{+}$head group; these results are consistent with the expected chargebalancing electrostatic interactions between the surfactant species and the MIF-1 framework. In the accompanying 1D ${ }^{13} \mathrm{C}\left\{{ }^{1} \mathrm{H}\right\}$ CPMAS spectrum, the two well-resolved ${ }^{13} \mathrm{C}$ signals at $149 \mathrm{ppm}$ and $151 \mathrm{ppm}$ indicate the presence of two inequivalent ${ }^{13} \mathrm{C}$ environments $\left(C_{3 \mathrm{a}}\right.$ and $\left.C_{3 \mathrm{~b}}\right)$ associated with the $\mathrm{sp}^{2}$ carbon atom bonded to the nitrogen atoms of the methylimidazole moieties. This is clearly evidenced in the 2D HETCOR spectrum, in which different intensity correlations are observed for these ${ }^{13} \mathrm{C}$ signals: while they both are correlated with the ${ }^{1} \mathrm{H}$ signal at $2.55 \mathrm{ppm}$ from the methyl protons of the methylimidazole group $\left(C_{4}\right)$, only the ${ }^{13} \mathrm{C}$ signal at $151 \mathrm{ppm}\left(C_{3 \mathrm{~b}}\right)$ is correlated with the ${ }^{1} \mathrm{H}$ signal at $2.45 \mathrm{ppm}$ from the $\mathrm{CTA}^{+}$head groups. This indicates that there are two distinct types of methylimidazole $C_{3}$ moieties, which differ according to their respective proximities to the $\mathrm{CTA}^{+}$head groups. Interestingly, the ${ }^{1} \mathrm{H}$ signal at $2.45 \mathrm{ppm}$ from the $\mathrm{CTA}^{+}$head groups is also correlated with the alkyl ${ }^{13} \mathrm{C}$ signals at $16 \mathrm{ppm}\left(C_{17^{\prime}}\right), 24 \mathrm{ppm}\left(C_{16^{\prime}}\right)$, and 31 ppm $\left(C_{15^{\prime}}\right)$ associated with the ends of the $\mathrm{CTA}^{+}$alkyl chains; these specific intensity correlations reflect an interdigitated arrangement of a fraction of $\mathrm{CTA}^{+}$molecules where the head group and tail are in close proximity to each other, an arrangement known from the surfactant crystal structure. For this short contact time, correlated signal intensity is also observed between the ${ }^{1} \mathrm{H}$ signal at $2.55 \mathrm{ppm}$ from the methyl protons of the methylimidazole moiety $\left(C_{4}\right)$ and the ${ }^{13} \mathrm{C}$ signal at $24 \mathrm{ppm}$ $\left(C_{16^{\prime}}\right)$ from the penultimate carbon atom on the end of the $\mathrm{CTA}^{+}$alkyl chain, which corroborates the interdigitated arrangement of some of the surfactant species. The molecular-level insights provided by these short-range intermolecular correlations (red arrows) are depicted in the schematic diagram in Fig. 5b.

For a longer contact time (5 ms), the $2 \mathrm{D}^{13} \mathrm{C}\left\{{ }^{1} \mathrm{H}\right\}$ HETCOR NMR spectrum (Fig. 5a, blue) acquired under otherwise identical 


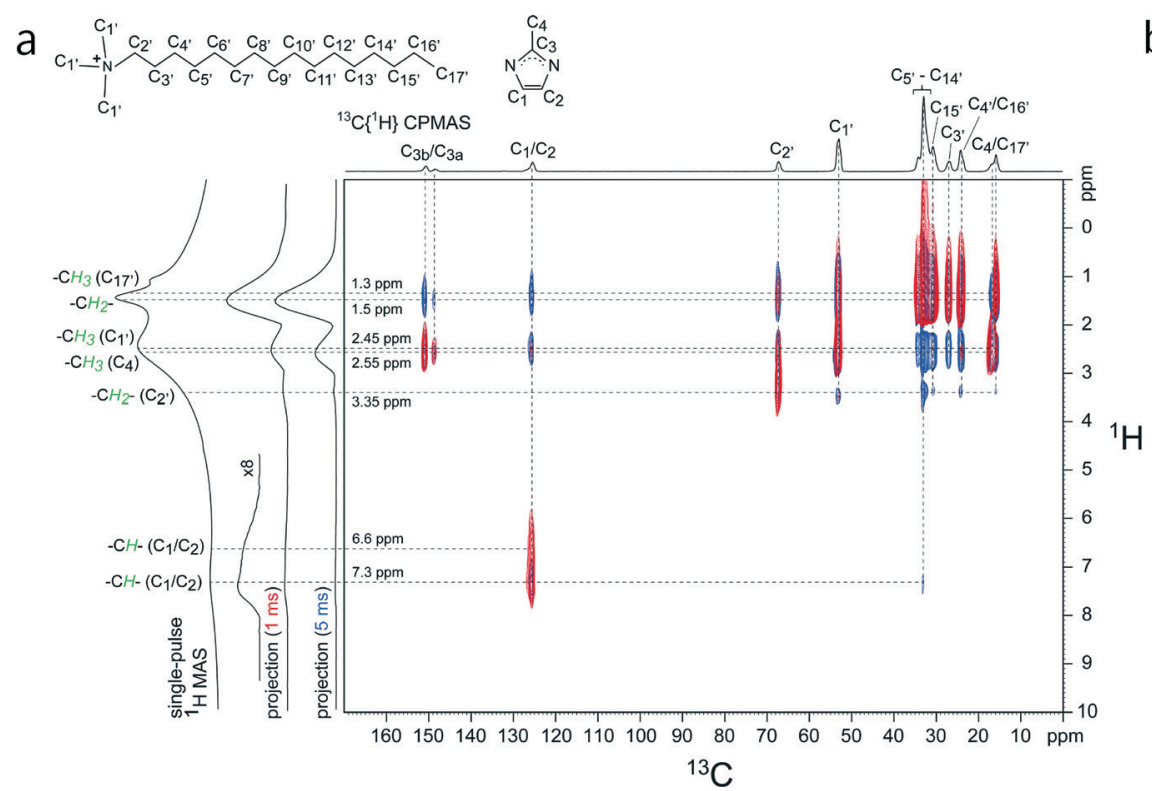

b

Fig. 5 (a) Solid-state $2 \mathrm{D}{ }^{13} \mathrm{C}\left\{{ }^{1} \mathrm{H}\right\}$ HETCOR NMR spectra acquired at room temperature for as-synthesized MIF-1 under MAS conditions of $12.5 \mathrm{kHz}$ with $1 \mathrm{~ms}$ (red) or $5 \mathrm{~ms}$ (blue) cross-polarization (CP) contact times. For comparison, 1D single-pulse ${ }^{1} \mathrm{H}$ MAS and $1 \mathrm{D}{ }^{13} \mathrm{C}\left\{{ }^{1} \mathrm{H}\right\} \mathrm{CPMAS}$ spectra are shown along the vertical and horizontal axes, respectively, of the 2D spectra. Schematic diagrams of the CTA ${ }^{+}$surfactant and methylimidazole molecules are shown and their corresponding ${ }^{13} \mathrm{C}$ and ${ }^{1} \mathrm{H}$ signal assignments are indicated in the $1 \mathrm{D}$ spectra. (b) Schematic diagram of MIF-1 ( $\mathrm{Zn} / \mathrm{MeIM} / \mathrm{CTAB}$ ) showing interactions between the zinc imidazolate chains and the $C T \mathrm{~A}^{+}$molecules that are consistent with the $2 \mathrm{D}{ }^{13} \mathrm{C}\left\{{ }^{1} \mathrm{H}\right\}$ HETCOR NMR analyses. Carbon and hydrogen atoms are labeled according to the assignments in Fig. 1. Red and blue arrows indicate ${ }^{13} \mathrm{C}-{ }^{1} \mathrm{H}$ dipolar interactions at short $(1 \mathrm{~ms})$ and long $(5 \mathrm{~ms})$ contact times, respectively. Note that $\mathrm{Zn}$ and $\mathrm{Br}$ atoms oriented into the page are colored gray. A fraction of the $\mathrm{CTA}^{+}$molecules are thought to be interdigitated with an antiparallel stacking arrangement.

conditions yields correlated signal intensity from more weakly coupled ${ }^{13} \mathrm{C}$ and ${ }^{1} \mathrm{H}$ nuclei (for reasons of internuclear distance or molecular mobilities). Intensity correlations are observed for the ${ }^{13} \mathrm{C}$ signal at $126 \mathrm{ppm}\left(C_{1} / C_{2}\right)$ with the ${ }^{1} \mathrm{H}$ signal at $1.3 \mathrm{ppm}$, revealing that the aromatic carbons $\left(C_{1} / C_{2}\right)$ of the methylimidazole are also molecularly proximate to the methyl protons of the $\mathrm{CTA}^{+}$tail. As observed and discussed above, the blue spectrum also reveals 2D signal intensity between both of the ${ }^{13} \mathrm{C}$ signals at $149 \mathrm{ppm}\left(C_{3 \mathrm{a}}\right)$ and $151 \mathrm{ppm}\left(C_{3 \mathrm{~b}}\right)$ and the ${ }^{1} \mathrm{H}$ signal at $1.5 \mathrm{ppm}$ associated with the $\mathrm{CTA}^{+}$alkyl protons, while only the $C_{3 \mathrm{~b}}$ carbon atom is molecularly close to the $\mathrm{CTA}^{+}$tail, based on the intensity correlation between the ${ }^{13} \mathrm{C}$ signal at $151 \mathrm{ppm}$ and the ${ }^{1} \mathrm{H}$ signal at $1.3 \mathrm{ppm}$. This difference between the two distinct signals for $C_{3}$ indicates a high degree of order in the material if the antiparallel arrangement of the surfactant chains is taken into account. These longer-range intermolecular correlations are depicted by the blue arrows in the schematic diagram of Fig. 5b.

Collectively, the NMR results offer complementary and additional insights to previous X-ray diffraction analyses of MIF-1, which characterize the long-range order of the materials, though are insensitive to structural disorder or order that is present only locally (e.g., several $\mathrm{nm}$ ). In particular, the absence of XRD reflections at high $2 \theta$ values - as opposed to the gemini-type MIFs - is consistent with a rather high degree of surfactant disorder commonly observed for other $\mathrm{CTA}^{+}$-directed mesostructured oxide materials. ${ }^{39,40}$ Nevertheless, the $2 \mathrm{D}^{13} \mathrm{C}\left\{{ }^{1} \mathrm{H}\right\}$ HETCOR NMR spectra establish that the head group moieties of the cationic $\mathrm{CTA}^{+}$surfactants interact strongly with the anionic zinc imidazolate framework, as previously observed for closely related $\mathrm{CTA}^{+}$-directed silicates with lamellar mesoscopic ordering. ${ }^{39,40}$ However, whereas the surfactant chains are highly disordered in the layered $\mathrm{CTA}^{+}$-silicate materials, MIF-1 has a significant fraction of its $\mathrm{CTA}^{+}$molecules that appear to be interdigitated between the zinc methylimidazolate chains with an anti-parallel stacking arrangement (Fig. 5b), as likewise inferred for the gemini-directed MIFs. Similar interdigitated, antiparallel arrangements of alkyl side chains on supramolecular assemblies of (linear) semiconducting polymers in bulk heterojunction materials have been observed, where the degree of ordering of the domains has a significant influence on the charge transfer properties. ${ }^{41,42}$ The discrepancy between the order observed in the 2D NMR experiments and apparent absence of order at the molecular level in the PXRD experiments can be explained in two ways: First, $\mathrm{CTA}^{+}$is an organic species that exhibits lower scattering factors compared to the inorganic layers, thus resulting in low scattering intensity especially at higher angles in the PXRD patterns. Secondly, the antiparallel arrangements of the alkyl side chains may be confined to small domains and broken up by significant conformational disorder, and therefore not detected by XRD methods.

To assess the importance of the halogenide counter-ions on the formation of lamellar MIF mesophases, the syntheses were conducted with gemini surfactant molecules without halogenide counter-ions (the bromine counter-ions were exchanged by nitrate ions via treatment with $\mathrm{AgNO}_{3}$ ). For all combinations of solvents and surfactant concentrations tested, 
no mesostructured products were formed in the absence of the halogenide counter-ions, under otherwise identical conditions. By comparison, syntheses conducted with chlorine counterions yielded MIF products (Fig. S7 $\dagger$ ). These results corroborate the important role of the halogenide counter-ions in syntheses of the MIF materials. In addition, the presence of the surfactant molecules appears to hinder the formation of ZIF structures, such as ZIF-8, which are typically obtained by mixing zinc nitrate and imidazole in methanol. ${ }^{43}$ However, adding halogenide ions to a reaction mixture containing methanol, zinc acetate, imidazole and surfactants with nitrate counter-ions led to the formation and rapid precipitation of a MIF product. Collectively, these observations underscore the importance of halogenide ions in the MIF syntheses.

Previously, a liquid crystal templating mechanism was proposed for the formation of MIF materials, according to which the mesostructure is directed by the surfactant molecules, while the coordination polymer nucleates and grows within the water reservoirs of the lamellar surfactant mesophase. ${ }^{20}$ As the curvature of the micellar structure formed by gemini surfactants is strongly dependent on the lengths of the tail and linker chains and is highly sensitive to the surfactant concentration and solvent composition, ${ }^{27,44}$ this formation mechanism should in principle result in a wide variety of mesostructures, which is not observed in the present case where exclusively lamellar nanomorphologies are obtained. We therefore propose a formation mechanism in which the one-dimensional structure composed of negatively charged $\left[\mathrm{Zn}(\mathrm{Me}) \mathrm{IMBr}_{2}\right]^{-}$chains is formed first. These then assemble with the positively charged gemini surfactants by an electrostatically driven cooperative assembly process that is similarly observed with various MCM-type systems, ${ }^{45}$ resulting in the 2D slab-like structures of the final lamellar mesostructured material (Fig. 6). Since the quasi-linear geometry of the zinc bromide imidazolate chains with vanishing curvature seems to direct and drive the mesostructure assembly, the formation of exclusively lamellar mesostructures with zero curvature is hence expected.

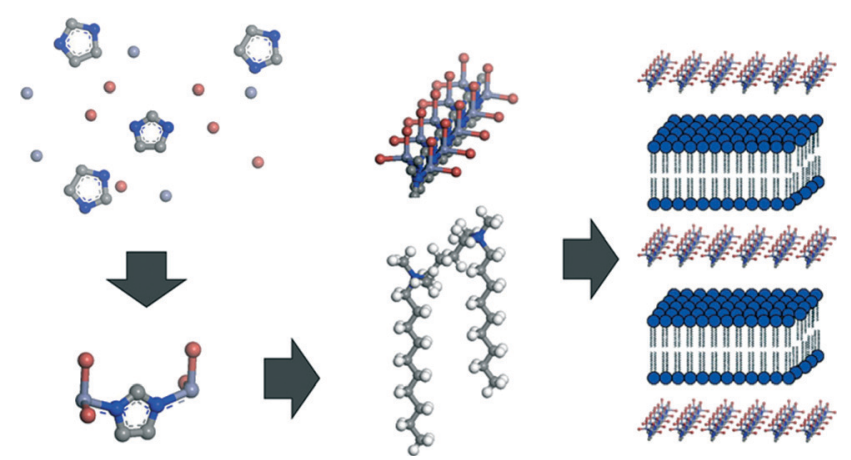

Fig. 6 Proposed formation of MIF materials by electrostatically-driven cooperative self-assembly. Upon addition of bromide to the solution containing imidazole, zinc and the surfactant, one-dimensional chains form and arrange into quasi two-dimensional layers, intercalated by surfactant ions for charge compensation. Note that the surfactants are omitted on the left-hand side for clarity.

\section{Conclusions}

The solid-state NMR analyses yield detailed atomic-level insights on the structures of metal imidazolate framework materials, which support previously proposed structural models. Specifically, surfactant bilayers between the layers of one-dimensional zinc-imidazolate chains charge-compensate the chain structure. Although our efforts to obtain cubic or hexagonal mesostructured MIFs have so far been unsuccessful, the proposed formation mechanism of the gemini-directed MIFs - electrostatically driven co-assembly governed by the formation of linear zinc bromide imidazolate chains - sheds light on why exclusively lamellar structures have been found so far in the MIF system. The roles of halogenide counterions in surfactant-assisted syntheses of MIF and ZIF materials are established, along with their influences on the selectivity and nanoscale morphologies of MIF versus ZIF products. These results have general implications for syntheses of mesostructured imidazolate framework materials and the molecular-level interactions that are responsible for the assembly and crystallization of their ordered frameworks.

\section{Acknowledgements}

Financial support from the Max Planck Society, the cluster of excellence "Nanosystems Initiative Munich" (NIM), the Center for NanoScience (CeNS), Chevron Energy Technology Company, and the U.S. National Science Foundation Graduate Research Fellowship under grant no. 1144085 are gratefully acknowledged. The solid-state 2D NMR experiments were conducted at the UCSB MRL Shared Experimental Facilities, which is supported by the MRSEC Program of the NSF under award no. DMR-1121053. We thank C. Minke, V. Duppel, and C. Ziegler for their assistance with the material analyses and an MPG-UCSB exchange program that led to this collaborative work.

\section{Notes and references}

1 J. L. C. Rowsell and O. M. Yaghi, Microporous Mesoporous Mater., 2004, 73, 3-14.

2 O. M. Yaghi, M. O'Keeffe, N. W. Ockwig, H. K. Chae, M. Eddaoudi and J. Kim, Nature, 2003, 423, 705-714.

3 W. Xuan, C. Zhu, Y. Liu and Y. Cui, Chem. Soc. Rev., 2012, 41, 1677-1695.

4 S.-L. Li and Q. Xu, Energy Environ. Sci., 2013, 6, 1656-1683.

5 O. K. Farha, I. Eryazici, N. C. Jeong, B. G. Hauser, C. E. Wilmer, A. A. Sarjeant, R. Q. Snurr, S. T. Nguyen, A. Ö. Yazaydın and J. T. Hupp, J. Am. Chem. Soc., 2012, 134, 15016-15021.

6 K. K. Tanabe and S. M. Cohen, Chem. Soc. Rev., 2011, 40, 498-519.

7 J. A. Thompson, N. A. Brunelli, R. P. Lively, J. R. Johnson, C. W. Jones and S. Nair, J. Phys. Chem. C, 2013, 117, 8198-8207.

8 J.-L. Wang, C. Wang and W. Lin, ACS Catal., 2012, 2, 2630-2640. 
9 R. Haldar, S. K. Reddy, V. M. Suresh, S. Mohapatra, S. Balasubramanian and T. K. Maji, Chem. - Eur. J., 2014, 20, 4347-4356.

10 H. Hosseini, H. Ahmar, A. Dehghani, A. Bagheri, A. Tadjarodi and A. R. Fakhari, Biosens. Bioelectron., 2013, 42, 426-429.

11 W. Ma, Q. Jiang, P. Yu, L. Yang and L. Mao, Anal. Chem., 2013, 85, 7550-7557.

12 S. Ma and H.-C. Zhou, Chem. Commun., 2010, 46, 44-53.

13 P. Cui, Y.-G. Ma, H.-H. Li, B. Zhao, J.-R. Li, P. Cheng, P. B. Balbuena and H.-C. Zhou, J. Am. Chem. Soc., 2012, 134, 18892-18895.

14 P. Horcajada, T. Chalati, C. Serre, B. Gillet, C. Sebrie, T. Baati, J. F. Eubank, D. Heurtaux, P. Clayette, C. Kreuz, J.-S. Chang, Y. K. Hwang, V. Marsaud, P.-N. Bories, L. Cynober, S. Gil, G. Ferey, P. Couvreur and R. Gref, Nat. Mater., 2010, 9, 172-178.

15 J. Della Rocca, D. Liu and W. Lin, Acc. Chem. Res., 2011, 44, 957-968.

16 H.-L. Jiang, Y. Tatsu, Z.-H. Lu and Q. Xu, J. Am. Chem. Soc., 2010, 132, 5586-5587.

17 N. Klein, I. Senkovska, K. Gedrich, U. Stoeck, A. Henschel, U. Mueller and S. Kaskel, Angew. Chem., Int. Ed., 2009, 48, 9954-9957.

18 B. Mu, P. M. Schoenecker and K. S. Walton, J. Phys. Chem. C, 2010, 114, 6464-6471.

19 X.-X. Huang, L.-G. Qiu, W. Zhang, Y.-P. Yuan, X. Jiang, A.-J. Xie, Y.-H. Shen and J.-F. Zhu, CrystEngComm, 2012, 14, 1613-1617.

20 S. C. Junggeburth, K. Schwinghammer, K. S. Virdi, C. Scheu and B. V. Lotsch, Chem. - Eur. J., 2012, 18, 2143-2152.

21 S. C. Junggeburth, L. Diehl, S. Werner, V. Duppel, W. Sigle and B. V. Lotsch, J. Am. Chem. Soc., 2013, 135, 6157-6164.

22 M. Choi, K. Na, J. Kim, Y. Sakamoto, O. Terasaki and R. Ryoo, Nature, 2009, 461, 246-249.

23 K. Na, M. Choi, W. Park, Y. Sakamoto, O. Terasaki and R. Ryoo, J. Am. Chem. Soc., 2009, 132, 4169-4177.

24 K. Na, C. Jo, J. Kim, K. Cho, J. Jung, Y. Seo, R. J. Messinger, B. F. Chmelka and R. Ryoo, Science, 2011, 333, 328-332.

25 F. M. Menger and C. A. Littau, J. Am. Chem. Soc., 1991, 113, 1451-1452.

26 F. M. Menger and C. A. Littau, J. Am. Chem. Soc., 1993, 115, 10083-10090.

27 R. Zana and Y. Talmon, Nature, 1993, 362, 228-230.
28 K. Karaborni, K. Esselink, P. A. J. Hilbers, B. Smit, J. Karthäuser, N. M. van Os and R. Zana, Science, 1994, 266, 254-256.

29 H. I. Lee, C. Pak, S. H. Yi, J. K. Shon, S. S. Kim, B. G. So, H. Chang, J. E. Yie, Y.-U. Kwon and J. M. Kim, J. Mater. Chem., 2005, 15, 4711-4717.

30 B. Elena, G. de Paëpe and L. Emsley, Chem. Phys. Lett., 2004, 398, 532-538.

31 Q. Huo, D. I. Margolese and G. D. Stucky, Chem. Mater., 1996, 8, 1147-1160.

32 K. Lund, N. Muroyama and O. Terasaki, Microporous Mesoporous Mater., 2010, 128, 71-77.

33 C. K. Lee, M. J. Ling and I. J. B. Lin, Dalton Trans., 2003, 4731-4737.

34 G. A. V. Martins, P. J. Byrne, P. Allan, S. J. Teat, A. M. Z. Slawin, Y. Li and R. E. Morris, Dalton Trans., 2010, 39, 1758-1762.

35 X. Ji, C. Liu, C. Liu, J. Li, X. Yu, P. Su, J. Huang, J. Jia, J. Wu, L. Chen, C. Chen, Y. Wu, Y. Fan, J. Lin, B. Cheng and C. Tang, Mater. Lett., 2014, 122, 309-311.

36 A. R. Campanelli and L. Scaramuzza, Acta Crystallogr., Sect. C: Cryst. Struct. Commun., 1986, 42, 1380-1383.

37 R. Zana, M. Benrraou and R. Rueff, Langmuir, 1991, 7, 1072-1075.

38 X. Yu, Z. Xu and S. Han, J. Porous Mater., 2010, 17, 99-105.

39 N. Hedin, R. Graf, S. C. Christiansen, C. Gervais, R. C. Hayward, J. Eckert and B. F. Chmelka, J. Am. Chem. Soc., 2004, 126, 9425.

40 S. C. Christiansen, D. Zhao, M. Janicke, C. C. Landry, G. D. Stucky and B. F. Chmelka, J. Am. Chem. Soc., 2001, $123,4519$.

41 A. C. Mayer, M. F. Toney, S. R. Scully, J. Rivnay, C. J. Brabec, M. Scharber, M. Koppe, M. Heeney, I. McCulloch and M. D. McGehee, Adv. Funct. Mater., 2009, 19, 1173.

42 N. C. Miller, E. Cho, M. J. N. Junk, R. Gysel, C. Risko, D. Kim, S. Sweetnam, C. E. Miller, L. J. Richter, R. J. Klinke, M. Heeney, I. McCulloch, A. Amassian, D. Acevedo-Feliz, C. Knox, M. R. Hansen, D. Dudenko, B. F. Chmelka, M. F. Toney, J. L. Brédas and M. D. McGehee, Adv. Mater., 2012, 24, 6071-6079.

43 H. Hayashi, A. P. Cote, H. Furukawa, M. O'Keeffe and O. M. Yaghi, Nat. Mater., 2007, 6, 501-506.

44 E. Buhler, E. Mendes, P. Boltenhagen, J. P. Munch, R. Zana and S. J. Candau, Langmuir, 1997, 13, 3096-3102.

45 R. Liu, Y. Shi, Y. Wan, Y. Meng, F. Zhang, D. Gu, Z. Chen, B. Tu and D. Zhao, J. Am. Chem. Soc., 2006, 128, 11652-11662. 